vol.4 No.1- 2017

\title{
DEEP VEIN THROMBOSIS IN CRITICALLY ILL TRAUMA PATIENTS: CLINICAL NURSING PROPHYLAXIS
}

\author{
Aida Faried Abd Elwanees Ali ${ }^{1}$, Mona Mohammed El Hady ${ }^{2}$, Amany \\ Mohamed Shebl ${ }^{3}$, Mohamed Ahmed Ahmed. Sultan ${ }^{4}$ \\ 1 Assistant lecturer, Faculty of Nursing, Mansoura University. \\ 2 Lecturer of Critical and Emergency Care Nursing, Faculty of Nursing, Mansoura University \\ 3 Professor of Medical Surgical Nursing, Faculty of Nursing, Mansoura University \\ 4 Professor of Anesthesia and Intensive Care, Faculty of Medicine, Mansoura University \\ E-mail of the corresponding author: aida_faried@yahoo.com
}

Abstract

Deep vein thrombosis (DVT) is a silent killer and it is the third most common vascular disease. Critically ill patients have general and specific risk factors for DVT. DVT prophylaxis is the most efficient and cost-effective way to prevent fatal and nonfatal VTE in critical illness. Critical care nurses play a key role in the detection and prevention of DVT. Thereby, this study aimed to evaluate the clinical nursing prophylaxis of deep vein thrombosis in critically ill trauma patients. Subjects and method a descriptive exploratory research design was utilized. The study was conducted on a convenience sample of fifty critical care nurses who worked in trauma intensive care units (ICUs) at Emergency Hospital, Mansoura University. Data were obtained using one tool; Nursing performance observational checklist for DVT preventive measures. This tool includes two parts; demographic characteristics of the nurses and DVT preventive measures observational check list. Results revealed that 62\% of nursing staff had 6-10 years of experience in ICU. It was found that $76 \%$ of the nursing staff graduated from secondary nursing school and $64 \%$ of them were in the age group of 30 years old and more with a mean age of (32.22 \pm 5.29). It was noted that $88 \%$ of the nursing staff in the intended ICUs did not attended inservice training course on preventive measures of DVT. It was observed that all of the nursing staff applied elastic stocking to the patients but their performance was less than $80 \%$ from the total steps of performance and more than half of them changed patient's position, performed the range of motion exercises (ROM) for the lower limbs and provided the deep breathing exercises with more than $80 \%$ of the performance steps. Also, all of the nursing staff instructed the patients about coughing exercises with more than $80 \%$ of the total performance steps, While $80 \%$ of them moved the patient on the chair with less than $80 \%$ of total performance. Conclusion The critical care nurses didn't provide a prophylaxis for DVT in critically ill trauma patients with a high performance level and there is need for continuing education to improve nurses' practices regarding DVT prophylaxis. The study recommended designing an education program and protocol about DVT prevention and it must be provided to health team members to be used as a reference guide in their performance. Integrating the DVT prevention protocol into care plan to replace the traditional nursing care plan.

Key words: Deep Vein Thrombosis, Nursing prophylaxis, prevention, intensive care unit, critically ill trauma patients

\section{Introduction:}

Venous thromboembolism (VTE) has to be a serious pathophysiologic condition that is a major cause of morbidity and mortality. A collective term, VTE describes a blood clot that forms inside the

vasculature and results in a DVT and/or a pulmonary embolism (PE). DVT and PE are considered the third leading cause of cardiovascular mortality, superseded only by myocardial infarction and stroke ${ }^{(\mathbf{1})}$. 
A deep vein thrombosis is defined as formation of blood clot in a deep vein of the legs such as femoral vein or popletial vein or deep veins of the pelvis and occasionally the veins of the arms are affected. The blood clot does not break down in a deep vein of the body. As the clot does not break down, it can become large and obstruct the normal flow of blood in the vein. Deep veins of the lower extremities are considered the most common sites for a DVT ${ }^{(2)}$.

Critically ill patients have similar general risk factors for DVT with other patients as advanced age, immobilization, obesity, past history of personal or familial VTE, past history of neoplasm, sepsis, stroke, respiratory or heart failure, pregnancy, trauma, or recent surgery. Mechanical ventilation and Central venous catheterization are considered one of the risk factors of DVT for critically ill patients ${ }^{(3)}$.

The Critical care nurse should be aware of DVT risk factors. Perform a thorough assessment for DVT risk factors and initiate DVT prophylaxis if risk is significant even with vague or missing symptoms especially the patients admitted to a critical care unit ${ }^{(4)}$.

Diagnosis and assessment for DVT are important, also appropriate prophylaxis and prevention when risk is present is the most effective way of avoiding DVT ${ }^{(5)}$.

Prevention of DVT and PE has become a major goal in the management of critically ill patients who are at high risk of DVT. Prevention of DVT is more effective in improving positive patient outcomes and is an important aspect of nursing care ${ }^{(6)}$.

Methods of prevention of DVT include a combination of mechanical and pharmacological prophylaxis. A variety of mechanical interventions are used as early mobilization, evaluate the patient's overall hydration and ensure compression stocking. Also bed rested patients need to be instructed to change position, dorsiflex their feet, and rotate their ankles every 2 to 4 hours. Any patients who are able to get out of bed should be in the chair for all meals and ambulate at least 3 times per day ${ }^{(7)}$.

No single technique can completely abolish DVT. Instead, a range of mechanical and pharmacological interventions may be used. Anticoagulants are used routinely for DVT prevention and treatment. The goal of anticoagulation therapy for DVT prophylaxis is to prevent DVT formation ${ }^{(8)}$.

Critical care nurses are in a key position to detect signs and symptoms of DVT promptly as well as to take measures to prevent DVT. The nurse should categorize the patient's risk as low, moderate, high and very high and she should tailor the DVT prophylaxis ${ }^{(9)}$.

The nurse should assess the patient's need for mechanical prophylaxis as elastic stocking. Also she should change the patient's position every two hours and elevate the legs of the patients about 10-20 degrees above the heart level. Early ambulation is an adjunct to the DVT prevention. It increases the venous return and reduces thrombosis. Active and passive range of motion exercises of the lower limbs should be encouraged for critically ill patients ${ }^{(\mathbf{1 0 , 1 1})}$.

The fluid balance should be maintained in the patients to avoid dehydration and hypercoagulability. Intake and output should be monitored every shift. Encourage fluid intake daily unless it is contraindicated. Determine baseline weight and daily weight. Also the nurse monitor the bowel status to avoid constipation and when patient has in active bowel movement, he may need a stool softener to avoid straining which may increase venous pressure on the lower extremities ${ }^{(\mathbf{1 2})}$. 
DEEP VEIN THROMBOSIS IN CRITICALLY ILL TRAUMA PATIENTS: etc...

\begin{abstract}
Significance of the study
The prevalence of DVT in critically ill patient is from $10 \%$ to $80 \%$ worldwide ${ }^{(\mathbf{1 0})}$. Patients in medical and surgical ICUs may have DVT within the first week of ICU admission. Approximately $60 \%$ of trauma patients exposed to DVT within the first 2 weeks of admission. About one third of patients with symptomatic DVT may develop a PE ${ }^{(\mathbf{1 3})}$.

Since DVT is a common complication of hospitalization for medical illnesses and surgical procedures. Also initiation of prophylaxis may be delayed, interrupted, or withheld because of risk of bleeding and the use of sequential compression devices and stockings have not been assessed rigorously in critically ill patients $(14,15)$. So this research was done to evaluate the clinical nursing prophylaxis of deep vein thrombosis in critically ill trauma patients.
\end{abstract}

\section{Subjects and Methods \\ Research design}

A descriptive research design was used to conduct this study.

\section{Research Setting:}

This study was carried out in the ICUs of Emergency Hospital, namely trauma ICU (trauma 1, trauma 2 and trauma 3) at Mansoura University Hospital.

\section{Subjects:}

A convenient sample of fifty critical care nurses who worked in the previously mentioned setting was included in this study.

Research Questions: What is the level of nurse's practice regarding DVT prophylaxis?

Tools and techniques of data collection:

One tool was used for data collection:

I. Nursing performance observational checklist for Deep vein thrombosis preventive measures: This tool was developed by the researchers after reviewing of the relevant literatures to assess and evaluate critical care nurses practices about DVT prevention in ICU. The tool was tested for validity and reliability. This tool includes two parts; demographic characteristics of the nurses and DVT preventive measures observational check list.

- Part I: "Demographic characteristics of the nurses". It includes nurse's years of experience, age, level of education, role of exercises performance and in-service education on DVT prevention.

-Part II: "Deep vein thrombosis preventive measures observational checklists". It includes six observational checklists for nursing procedures that are considered to be effective in prevention of DVT. These checklists included applying elastic stocking checklist, changing position in the bed checklist, deep breathing and coughing exercises checklists, ROM for lower limbs checklist and transferring conscious patient from bed to chair checklist.

\section{Method}

This study was conducted on two phases; preparation and assessment phases.

\section{Preparatory phase}

- Ethical consideration approval was obtained from the research ethics committee of the Faculty of Nursing Mansoura University.

- Tool was developed by the researchers based on review of the recent related literatures.

- The reliability of the tool was measured using alpha Cronbach's test and the values of alpha Cronbach's were (0.959).

- The tool was tested for content validity by 5 experts in the field of Critical Care \& Emergency Nursing and Critical Care Medicine from the Faculties of Nursing and Medicine.

- Approval permission to conduct the study was obtained from the hospital 
administrative personnel based on the official letter after explanation of the aim and nature of the study.

- Informed consent was obtained from the nurses before the beginning of the study after explanation the purpose of the study, privacy and confidentiality of the collected data were assured and participants were able to withdraw from the study at any stage without responsibility.

- A pilot study was carried out on 5 nurses (10\% of sample size) who were excluded from the study subjects to test the feasibility, clarity and applicability of the tools, and the necessary modifications were done accordingly.

\section{Assessment phase:}

- During this stage the researchers assessed the demographic characteristics of nurses by using part one of tool I. Then observed the actual nursing performance of DVT prevention measures by using the part II of tool one once per shift for three shifts (morning, afternoon and evening shift) per each studied nurse.

\section{Ethical considerations}

- Ethical consideration approval was obtained from Scientific Research Ethics Committee of the Faculty of Nursing-Mansoura University.

- The researcher explained to the participants that there was no risk or hazards related to the study.

- Confidentiality of each subject were assured through the collected data were assured through coding of all data.

- Each participant was informed that his participation in the study was voluntary and they could withdraw at any stage without any responsibility.

\section{Statistical analysis}

After data were collected and transferred into special design formats so as to be suitable for computer feeding, data were analyzed using PC with Statistical
Package for Social Sciences (SPSS) version 16.0 .

Data were presented using descriptive statistics in the form of frequencies and percentages for qualitative variables, and means and standard deviations for quantitative variables. Collected data were statistically analyzed, presented in tables and used appropriate reliable, valid statistical methods \& tests.

\section{Results:}

Table (1): demonstrates the demographic characteristics of the critical care nurses working in the critical care units. The total numbers of the nursing staff who provide direct care for the critically ill trauma patients in the studied ICU were 50 nurses. It can be noted from this table that $62 \%$ of nursing staff had 610 years of experience in ICU.

Furthermore it was found that $76 \%$ of the nursing staff graduated from secondary nursing school and $64 \%$ of them were in the age group of 30 years old and more with a mean age of $(32.22 \pm 5.29)$ and the physiotherapy for the patients was performed by those nurses only. Also it was noted that $88 \%$ of the nursing staff in the intended ICUs did not attended inservice training course on preventive measures of DVT.

Table (2): reveals number and percentage of nurses' practice regarding elastic stocking. It was observed that all nursing staff didn't assess the patient's need for elastic stocking, clean leg, apply small amount of talcum powder to legs and feet or make documentation of elastic stocking practice.

Table (3): demonstrates number and percentage of nurses' practice regarding changing patient's position. It was observed that all nursing staff didn't perform the assessment and preparation practice to change patient's position. While, $68 \%$ of them was performed the implementation and documentation practices to change patient position. 


Table (4): shows number and
percentage of nurses' practice regarding
deep breathing exercises. It was observed
that all of the nursing staff didn't perform
the assessment practice of deep breathing
exercises. Also, only $\mathbf{6 8 \%}$ of them did the
preparation and implementation practices
of this procedure to the patients. Moreover
it was observed that all of the nurses didn't
make documentation of this procedure.

Table (5): reveals number and percentage of nurses' practice regarding coughing exercises. It was observed that all of the nursing staff didn't assess patient's cardiopulmonary status. Also it was noted that $60 \%$ of them didn't wash hands, explain procedure and put patient in sitting position. On the other side, all nurses instructed patients to apply cough exercise and they were document the practice's date and time, frequency, patient's cardiopulmonary status and reaction.

Table (6): explains number and percentage of nurses' practice regarding range of motion exercises for the lower limbs. It was observed that $\mathbf{6 0 \%}$ of the nursing staff assessed patient's physical status. Sixty percent of the nurses was not performed the preparation steps of this procedure. Moreover, only $\mathbf{7 8} \%$ of them was performed the implementation steps of lower limb range of motion exercises. While, $\mathbf{8 0 \%}$ of the nurses didn't perform documentation for this practice.

Table (7): explore number and percentage of nurses' practice regarding moving conscious patient to the chair. It was observed that $\mathbf{8 0 \%}$ of the nursing staff didn't perform the assessment practice for moving conscious patient to the chair.
Sixty percent of the nurses were explained procedure to the patient and elevated bed. Moreover, $\mathbf{8 0 \%}$ of the nurses didn`t perform the implementation practice to moving conscious patients outside the beds. While, all nurses didn't perform documentation practices for this procedure.

Table (8) and figure (1): states the mean nurses' practice score regarding DVT prophylaxes. It was mentioned that mean value for practice of elastic stocking was $(9.60 \pm 0.782)$ and the mean value for practice of changing the patient positioning was (11.68 \pm 0.47$)$. Also, it was noted that mean value for practice of Deep breathing exercise was $(4.04 \pm 1.41)$ and mean value for practice of coughing exercise was (2.00 \pm 0.00$)$. Moreover it was noticed from this table that the mean value for ROM exercise practice was $(15.60 \pm 2.50)$ and the mean value for moving the conscious to the chair practice

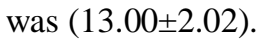

Figure (2): reveals the total nurses performance score for DVT preventive measures. It was observed that all of the nursing staff applied elastic stocking to the patients but their performance was less than $80 \%$ from the total steps of performance and more than half of them changed patient's position, performed the ROM for the lower limbs and provided the deep breathing exercises with more than $80 \%$ of the performance steps. Also, all of the nursing staff instructed the patients about coughing exercises with more than $80 \%$ of the total performance steps, While $80 \%$ of them moved the patient on the chair with less than $80 \%$ of total performance. 
Aida Faried Abd Elwanees Ali et. al.

Table 1: Demographic characteristics of the studied critical care nurses.

\begin{tabular}{|l|l|l|l|}
\hline \multicolumn{2}{|c|}{ Characteristics } & Number & \% \\
\hline \multirow{3}{*}{ Age in years } & $<30 \mathrm{y}$ & 18 & 36.0 \\
\cline { 2 - 4 } & $\geq 30 \mathrm{y}$ & 32 & 64.0 \\
\cline { 2 - 4 } & Mean \pm SD & $32.22 \pm 5.29$ \\
\hline Years of experience in ICU & $2-5$ & 17 & 34 \\
\cline { 2 - 4 } & $6-10$ & 31 & 62 \\
\cline { 2 - 4 } & $>10$ & 2 & 4.0 \\
\hline \multirow{2}{*}{ Level of education } & Secondary nursing school & 38 & 76 \\
\cline { 2 - 4 } & Bachelor & 12 & 24 \\
\hline \multirow{3}{*}{ Physiotherapy duties } & No & 0 & 0.0 \\
\cline { 2 - 4 } & Yes & 50 & 100 \\
\hline \multirow{2}{*}{$\begin{array}{l}\text { Attending in-service training course } \\
\text { on preventive measures of DVT }\end{array}$} & Yes & 6 & 12 \\
\cline { 2 - 4 } & No & 44 & 88 \\
\hline
\end{tabular}

Table 2: Number and percentage of nurses' practice regarding elastic stocking

\begin{tabular}{|c|c|c|c|c|}
\hline \multirow{2}{*}{ Nurse's practice } & \multicolumn{2}{|c|}{ Done } & \multicolumn{2}{|c|}{ Not done } \\
\hline & No & $\%$ & No & $\%$ \\
\hline \multicolumn{5}{|l|}{ Assessment } \\
\hline 1. Assess need for stocking. & 0 & 0.0 & 50 & 100 \\
\hline 2. Obtain physician's order. & 50 & 100 & 0 & 0.0 \\
\hline 3. Assess patient's skin and circulation. & 50 & 100 & 0 & 0.0 \\
\hline 4. Explain procedure. & 50 & 100 & 0 & 0.0 \\
\hline 5. Measure calf circumference. & 50 & 100 & 0 & 0.0 \\
\hline \multicolumn{5}{|l|}{ Preparation } \\
\hline 1) Wash hands. & 50 & 100 & 0 & 0.0 \\
\hline 2) Place patient in supine position. & 50 & 100 & 0 & 0.0 \\
\hline 3) Clean leg and put talcum powder. & 0 & 0.0 & 50 & $\mathbf{1 0 0}$ \\
\hline \multicolumn{5}{|l|}{ Implementation } \\
\hline 1) Apply stocking. & 50 & 100 & 0 & 0.0 \\
\hline 2) Reposition patient in comfort position. & 50 & 100 & 0 & 0.0 \\
\hline 3) Wash hands. & 50 & 100 & 0 & 0.0 \\
\hline 4) Inspect stocking. & 50 & 100 & 0 & 0.0 \\
\hline 5) Remove stockings at once shift. & 0 & 0.0 & 50 & $\mathbf{1 0 0}$ \\
\hline \multicolumn{5}{|l|}{ Documentation } \\
\hline 6) Recording the procedure. & 0 & 0.0 & 50 & $\mathbf{1 0 0}$ \\
\hline
\end{tabular}


DEEP VEIN THROMBOSIS IN CRITICALLY ILL TRAUMA PATIENTS: etc...

\begin{tabular}{|c|c|c|c|c|}
\hline \multirow{2}{*}{ Nurse's practice } & \multicolumn{2}{|c|}{ Done } & \multicolumn{2}{|c|}{ Not done } \\
\hline & No & $\%$ & No & $\%$ \\
\hline \multicolumn{5}{|l|}{ Assessment } \\
\hline 1. Assess cardiopulmonary status. & 0 & 0.0 & 50 & 100 \\
\hline \multicolumn{5}{|l|}{ Preparation } \\
\hline 1. Explain procedure. & 0 & 0.0 & 50 & 100 \\
\hline 2. Wash hand & 0 & 0.0 & 50 & 100 \\
\hline 3. Elevate bed level. & 0 & 0.0 & 50 & 100 \\
\hline \multicolumn{5}{|l|}{ Implementation } \\
\hline 1. Move the patient to the bed side. & 34 & 68 & 16 & 32 \\
\hline 2. Place one of the nurse feet in front of the other. & 34 & 68 & 16 & 32 \\
\hline 3. Place one arm under the patient's shoulder. & 34 & 68 & 16 & 32 \\
\hline 4. Place one hand under his hips. & 34 & 68 & 16 & 32 \\
\hline $\begin{array}{l}\text { 5. Move him toward other side to turn the patient to } \\
\text { his left side. }\end{array}$ & 34 & 68 & 16 & 32 \\
\hline $\begin{array}{l}\text { 6. To turn the patient to the right side cross the left } \\
\text { leg over the right. }\end{array}$ & 34 & 68 & 16 & 32 \\
\hline 7. Place the patient's arm across the chest. & 34 & 68 & 16 & 32 \\
\hline 8. Follow steps 7 and8. & 34 & 68 & 16 & 32 \\
\hline 9. Move him toward other side. & 34 & 68 & 16 & 32 \\
\hline 10. Turning the patient to his right side. & 34 & 68 & 16 & 32 \\
\hline 11. Frequency of repositioning every $2 \mathrm{hrs}$. & 34 & 68 & 16 & 32 \\
\hline \multicolumn{5}{|l|}{ Documentation } \\
\hline 1. Recording the procedure. & 34 & 68 & 16 & 32 \\
\hline
\end{tabular}

Table 4: Number and percentage of nurses' practice regarding deep breathing exercises:

\begin{tabular}{|c|c|c|c|c|}
\hline \multirow{2}{*}{ Nurse's practice } & \multicolumn{2}{|c|}{ Done } & \multicolumn{2}{|c|}{ Not done } \\
\hline & No & $\%$ & No & $\%$ \\
\hline \multicolumn{5}{|l|}{ Assessment } \\
\hline 1. Assess cardiopulmonary status. & 0 & 0.0 & 50 & 100 \\
\hline \multicolumn{5}{|l|}{ Preparation } \\
\hline 1. Wash hands. & 34 & 68 & 16 & 32 \\
\hline 2. Explain procedure. & 34 & 68 & 16 & 32 \\
\hline \multicolumn{5}{|l|}{ Implementation } \\
\hline 1. Position the patient with knee bent. & 34 & 68 & 16 & 32 \\
\hline 2. Place the left hand on the thoracic cage. & 34 & 68 & 16 & 32 \\
\hline 3. $\quad$ Place the right hand over the umbilicus. & 34 & 68 & 16 & 32 \\
\hline 4. Instruct the patient to turn his head to opposite side. & 34 & 68 & 16 & 32 \\
\hline 5. Instruct the patient to take a deep breath. & 34 & 68 & 16 & 32 \\
\hline $\begin{array}{l}\text { 6. Instruct the patient to exhale via pursed lips, mouth, } \\
\text { pulling on and contracting abdominal muscle. }\end{array}$ & 34 & 68 & 16 & 32 \\
\hline 7. Frequency of deep breathing exercises every $2 \mathrm{hrs}$. & 0 & 0.0 & 50 & 100 \\
\hline \multicolumn{5}{|l|}{ Documentation } \\
\hline 1. Recording the procedure. & 0 & 0.0 & 50 & 100 \\
\hline
\end{tabular}


Aida Faried Abd Elwanees Ali et. al.

Table 5: Number and percentage of nurses' practice regarding coughing exercises:
\begin{tabular}{|l|l|l|l|l|}
\hline \multicolumn{1}{|c|}{ Nurse's practice } & \multicolumn{2}{|c|}{ Done } & \multicolumn{2}{c|}{ Not done } \\
\cline { 2 - 5 } & No & $\%$ & \multicolumn{1}{|c|}{ No } & $\%$ \\
\hline Assessment & 0 & 0.0 & 50 & 100 \\
\hline 1. Assess cardiopulmonary status. & 20 & 40 & 30 & 60 \\
\hline Preparation & 20 & 40 & 30 & 60 \\
\hline 1. Wash hands. & 20 & 40 & 30 & 60 \\
\hline 2. Explain procedure. & \multicolumn{5}{|l|}{} \\
\hline 3. Position the patient in the bed with knee bent. & 50 & 100 & 0 & 0.0 \\
\hline Implementation & 0 & 0.0 & 50 & 100 \\
\hline 1. Instruct the patient to take a deep breath and then coughs & \multicolumn{5}{|l|}{} \\
\hline 2. Frequency of coughing exercises every 2hrs. & 50 & 100 & 0 & 0.0 \\
\hline Documentation & 1. Recording the procedure.
\end{tabular}

Table 6: Number and percentage of nurses' practice regarding range of motion exercises for the lower limbs.

\begin{tabular}{|c|c|c|c|c|}
\hline \multirow{2}{*}{ Nurse's practice } & \multicolumn{2}{|c|}{ Done } & \multicolumn{2}{|c|}{ Not done } \\
\hline & No & $\%$ & No & $\%$ \\
\hline \multicolumn{5}{|l|}{ Assessment } \\
\hline 1- Assess physical status. & 30 & 60 & 20 & 40 \\
\hline \multicolumn{5}{|l|}{ Preparation } \\
\hline 1. Wash hands. & 20 & 40 & 30 & 60 \\
\hline 2. $\quad$ Explain procedure. & 20 & 40 & 30 & 60 \\
\hline \multicolumn{5}{|l|}{ Implementation } \\
\hline \multicolumn{5}{|l|}{ 1- Hip ( ball and socket joint ) } \\
\hline a. Flexion. & 39 & 78 & 11 & 22 \\
\hline b. Extension. & 39 & 78 & 11 & 22 \\
\hline c. Hyperextension. & 39 & 78 & 11 & 22 \\
\hline d. Abduction. & 39 & 78 & 11 & 22 \\
\hline e. Adduction. & 39 & 78 & 11 & 22 \\
\hline f. $\quad$ Circumduction. & 39 & 78 & 11 & 22 \\
\hline g. Internal rotation. & 39 & 78 & 11 & 22 \\
\hline h. External rotation. & 39 & 78 & 11 & 22 \\
\hline \multicolumn{5}{|l|}{ 2- Knee ( hinge joint ) } \\
\hline a. Flexion. & 39 & 78 & 11 & 22 \\
\hline b. Extension. & 39 & 78 & 11 & 22 \\
\hline \multicolumn{5}{|l|}{ 3- Ankle ( hinge joint ) } \\
\hline a. Planter flexion. & 39 & 78 & 11 & 22 \\
\hline b. Dorsiflexion. & 39 & 78 & 11 & 22 \\
\hline \multicolumn{5}{|l|}{ 4- Foot ( gliding joint ) } \\
\hline a. Eversion. & 39 & 78 & 11 & 22 \\
\hline b. Inversion. & 39 & 78 & 11 & 22 \\
\hline \multicolumn{5}{|l|}{ 5- Toes ( condyloid ) } \\
\hline a. Flexion. & 39 & 78 & 11 & 22 \\
\hline b. Extension. & 39 & 78 & 11 & 22 \\
\hline c. Abduction. & 39 & 78 & 11 & 22 \\
\hline $\begin{array}{l}\text { d. Frequency of changing positions every } \\
\text { 2hrs. }\end{array}$ & 0 & 0.0 & 50 & 100 \\
\hline \multicolumn{5}{|l|}{ Documentation } \\
\hline 1. Recording the procedure. & 10 & 20 & 40 & 80 \\
\hline
\end{tabular}


DEEP VEIN THROMBOSIS IN CRITICALLY ILL TRAUMA PATIENTS: etc...

Table 7: Number and percentage of nurses' practice regarding moving conscious patient to the chair

\begin{tabular}{|c|c|c|c|c|}
\hline \multirow{2}{*}{ Nurse's practice } & \multicolumn{2}{|c|}{ Done } & \multicolumn{2}{|c|}{ Not done } \\
\hline & No & $\%$ & No & $\%$ \\
\hline \multicolumn{5}{|l|}{ Assessment } \\
\hline 1. Measure pulse rate. & 10 & 20 & 40 & 80 \\
\hline \multicolumn{5}{|l|}{ Preparation } \\
\hline 2. Explain procedure. & 30 & 60 & 20 & 40 \\
\hline 3. Bring bed to low position. & 30 & 60 & 20 & 40 \\
\hline \multicolumn{5}{|l|}{ Implementation } \\
\hline 4. Place chair or wheelchair beside the bed. & 10 & 20 & 40 & 80 \\
\hline 5. Lock wheels of wheelchair. & 10 & 20 & 40 & 80 \\
\hline 6. Support patient back with one hand. & 10 & 20 & 40 & 80 \\
\hline 7. Move patient's legs to side of bed. & 10 & 20 & 40 & 80 \\
\hline $\begin{array}{l}\text { 8. Allow patient to sit momentarily in this } \\
\text { position. }\end{array}$ & 10 & 20 & 40 & 80 \\
\hline 9. Place shoes or slippers on his feet. & 10 & 20 & 40 & 80 \\
\hline 10. Stand in front of the patient. & 10 & 20 & 40 & 80 \\
\hline 11. Place your hand around the patient's waist. & 10 & 20 & 40 & 80 \\
\hline $\begin{array}{l}\text { 12. Press your knees against his knees and lift } \\
\text { him upward. }\end{array}$ & 10 & 20 & 40 & 80 \\
\hline 13. Lower the person gradually into the chair. & 10 & 20 & 40 & 80 \\
\hline 14. Position patient on the chair. & 10 & 20 & 40 & 80 \\
\hline $\begin{array}{l}\text { 15. Frequency of moving conscious patient } \\
\text { twice per shift for } 10 \text { minutes. }\end{array}$ & 0 & 0.0 & 50 & 100 \\
\hline \multicolumn{5}{|l|}{ Documentation } \\
\hline 1. Evaluate the patient comfort and safety. & 0 & 0.0 & 50 & $100 \%$ \\
\hline $\begin{array}{l}\text { 2. Measure pulse } 10 \text { seconds after sitting on } \\
\text { the chair. }\end{array}$ & 0 & 0.0 & 50 & $100 \%$ \\
\hline 2. $\quad$ Recording the procedure. & 0 & 0.0 & 50 & $100 \%$ \\
\hline
\end{tabular}

Table 8: Mean nurses practice score regarding DVT prophylaxes

\begin{tabular}{|l|c|c|}
\hline \multicolumn{1}{|c|}{ Nurse's practice } & X \pm SD & Min-Max \\
\hline Elastic stocking & $9.60 \pm 0.782$ & $7-10$ \\
\hline Changing the patient positioning & $11.68 \pm 0.47$ & $11-12$ \\
\hline Deep breathing exercise & $4.04 \pm 1.41$ & $2-5$ \\
\hline Coughing exercise & $2.00 \pm 0.00$ & $2-2$ \\
\hline ROM exercise & $15.60 \pm 2.50$ & $12-19$ \\
\hline Moving the conscious patient to the chair & $13.00 \pm 2.02$ & $12-17$ \\
\hline Total practice score & $55.92 \pm 3.96$ & $48-65$ \\
\hline
\end{tabular}




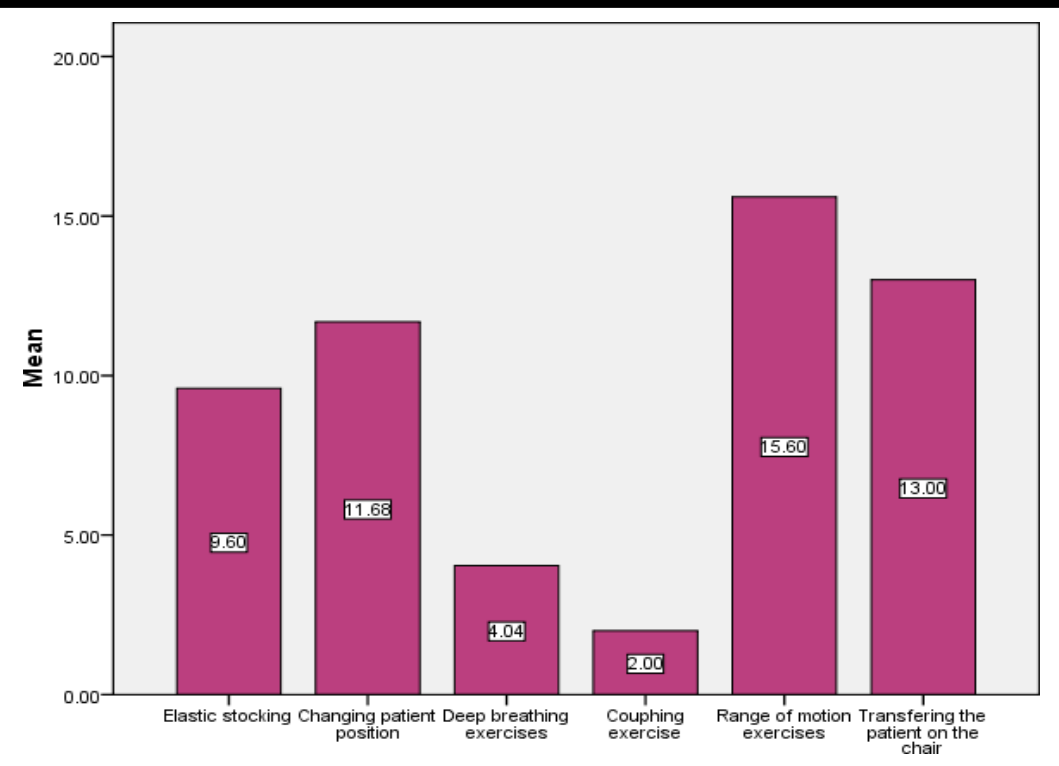

Figure 1: Mean nurses practice score regarding DVT prophylaxes

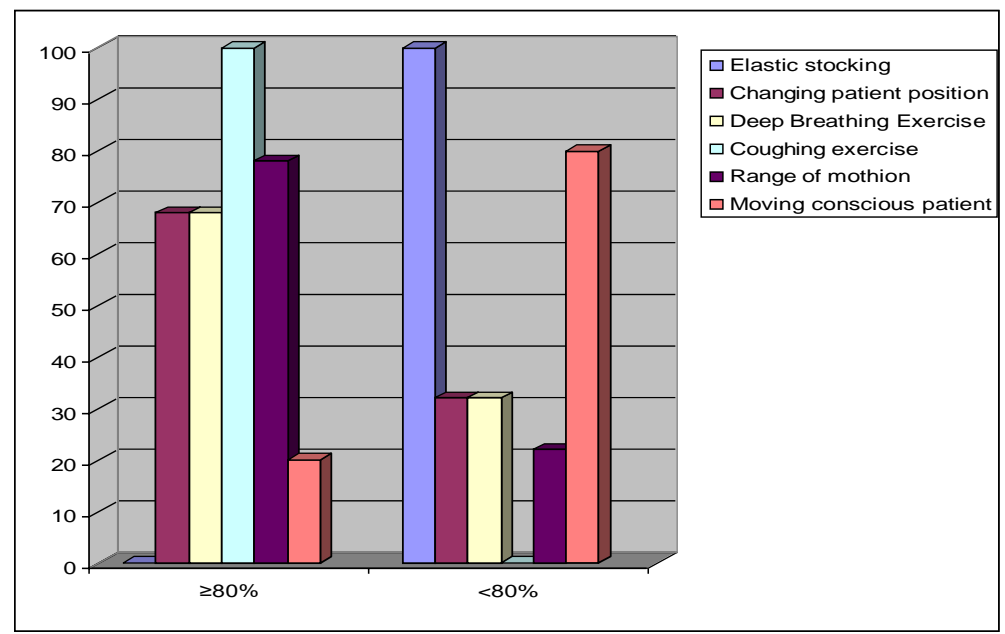

Figure 2: Total performance of critical care nurses for deep vein thrombosis preventive measures (less than $80 \%$ and more than $80 \%$ )

\section{Discussion:}

Deep Vein Thrombosis is a common and severe complication of critical illness and it is frequently unrecorded in critically ill patients and yet its complications lead to high morbidity and mortality associated with critical illness. DVT remains preventable complications, especially in critically ill patients after the advances of the prophylaxis (Fries, 2011; Minet et al., 2015).

Critical care nurses are in a key position to detect signs and symptoms of DVT promptly as well as to take measures to prevent DVT. An awareness of the 
patients at high risk of DVT or PE is vital.

The nurse should categorize the patient's risk as low, moderate, high and very high on the basis of pervious information and she should tailor the DVT prophylaxis (Anthony, 2013).

In the current study the researcher assessed the demographic characteristics of the critical care nurses and observed routinely DVT preventive measures. Regarding the demographic characteristics of the studied critical care nurses, it was noted that the majority of nursing staff had 6-10 years of experience in ICU and more than seventy percent of nursing staff graduated from secondary nursing school and $64 \%$ of them were in the age group of 30 years old and more with a mean age of $(32.22 \pm 5.29)$

Regarding the attendance of inservice training course, it was observed in the present study that majority of the nursing staff did not attended in-service training course on preventive measures of DVT however there is a greatest need for increasing the nurses awareness of DVT risk factors, diagnosis and chemical and mechanical prevention. This result is on the same line with Songwathana et al. (2010) who reported that only 3 nurses (7.14\%) had training or had attended a seminar on DVT.

In relation to the number and percentage of nurses' practice regards elastic stocking. It was observed that all nursing staff didn't assess the patient's need for elastic stocking, clean leg, apply small amount of talcum powder to legs and feet or make documentation of elastic stocking practice. This low level of practice may be resulting from lack of education concerning critical care nursing.
In this respect $(\mathbf{K i m} \&$ Lee,

2014) recorded that there are practical difficulties hindering appropriate use of the preventive measures for DVT as graduated compression stocking and intermittent pneumatic compression for critically ill patients and these difficulties due to lack of the systematic education based on the nurses needs.

As regards to number and percentage of nurses' practice regards change the patient's position. It was observed that all nursing staff didn't perform the assessment and preparation practice to change patient's position. While, the third of them was performed repositioning every $2 \mathrm{hrs}$. The reason related to in adequate repositioning is lack of training on this technique. This result is in harmony with Goldhill et al., (2008) who mentioned that only about third of study sample turned the patients every 2 hours.

In relation to number and percentage of nurses' practice regarding deep breathing exercises. It was observed that all of the nursing staff didn't perform the assessment practice of deep breathing exercises. Also, only more than the half of them did the preparation and implementation practices of this procedure to the patients. Moreover it was observed that all of the nurses didn't make documentation of this procedure. This variation of level of practice may be due to lack of training concerning chest physiotherapy and absence of respiratory therapists in studied ICUs. This finding is in agreement with Baidya et al., (2016) who reported that there was lack of training of physiotherapy practice in ICU and physiotherapists are not practicing 
deep breathing exercise training to a great extent.

Concerning the number and percentage of nurses' practice regards coughing exercises. It was observed that all of the nursing staff didn't assess patient's cardiopulmonary status. Also it was noted that sixty percent of them didn't wash hands, explain procedure and put patient in sitting position.

The reason of these findings may be due to lack in experience and updated knowledge concerning the ICU procedures. This result are supported by Yeole et al., (2015) who showed that physiotherapy provider in ICUs surveyed lack in experience and updated knowledge and there no exists established criteria for deep breathing and coughing treatment in ICU.

In relation to number and percentage of nurses' practice regards ROM exercises for the lower limbs. It was observed that more than half of the nursing staff assessed patient's physical status. Most of the nurses were not performed the preparation steps of this procedure. Moreover, only more than tow thirds of them were performed the implementation steps of lower limb ROM exercises. While, the majority of the nurses was not performed documentation for this practice. From this result it was noticed that ROM exercises can be possible to implement and these results are in the same way with Songwathana et al. (2010) who mentioned that regarding nursing interventions to prevent DVT it was found that promoting range of motion exercise was identified by most nurses $(97.62 \%)$ as having the greatest possibility of implementation.
Concerning nurses' practice regards moving conscious patient to the chair. It was observed that majority of the nursing staff didn't perform the assessment practice for moving conscious patient to the chair. Sixty percent of the nurses were explained procedure to the patient and elevated bed. Moreover, majority of the nurses didn't perform the implementation practice to moving conscious patients outside the beds. While, all nurses were not performed documentation practices of this procedure. The inadequate level of this practice may be due to lack of protocols, guideline and lack of education on mobilization of critically ill patients to the chair.

In the same line Songwathana et al. (2010) mentioned that some activities of DVT prevention reported with less than $90 \%$ of implementation as encouraging patients to ambulate (get out of bed) and $t$ the use of evidence-based clinical practice guidelines for deep vein thrombosis prevention could enhance early detection for DVT and maintaining blood flow velocity so patients can be able to get out of the bed.

Also Dafoe et al., (2015) mentioned that insufficient education considers barrier to mobilization to passive or active transfer to chair in the hospital ICU and there should be strategies to overcome them. Moreover Jesus et al., (2015) explained that the multidisciplinary team should prioritize the patient mobility status in ICU and should not underestimate the capacity of mobility of patients out of the bed.

As regards to total performance of critical care nurses for DVT preventive measures, it was observed in the current 
DEEP VEIN THROMBOSIS IN CRITICALLY ILL TRAUMA PATIENTS: etc...

study that all nurses applied elastic stocking to patients as a preventive measures of DVT but their performance was less than eighty percent from the total steps of performance and more than half of the nurses changed patient's position and teaching the deep breathing exercises with more than eighty percent of the performance steps.

Also all of the nursing staff instructed the patients about coughing exercises with more than eighty percent of the performance steps. Moreover seventy percent of the critical care nurses performed the range of motion exercises for the lower limbs for the patients with more than eighty percent of the total performance steps, while the majority of them transferred the conscious patient on the chair with less than eighty percent from the total steps of performance.

These variations in the performance of preventive measures of DVT are due to differences in the nurse's qualification where in the current study there was variation of qualification of nurses graduated from faculty of nursing and secondary nursing school. Also this variation could be attributed to limited years of experience of the nurses being less than 5 years in addition to lack of the ongoing education and nursing care updates on prophylaxis of DVT in critically ill patients.

These findings agreed with Songwathana et al. (2010) who reported that only 3 nurses $(7.14 \%)$ had training or had attended a seminar on DVT and it was affect on the nursing practice for prevention of DVT and they mentioned that regarding nursing interventions to prevent DVT it was found that promoting range of motion exercise was identified by most nurses (97.62\%) as having the greatest possibility of implementation. However, other activities reported with less than $90 \%$ of implementation included encouraging patients to ambulate (get out of bed) and applying compression. For recording of nursing interventions to prevent DVT, it recorded by all nurses (>90\%).

So it is clear to say that nursing education on DVT prevention interventions is very important in our critical care setting. This opinion is supported by Bonner \& Johnson (2014) who believed that nurses need to have appropriate skills and knowledge about the signs and symptoms of DVT, common diagnostic tests, pharmacological and mechanical treatments, and the follow up investigations offered to the patients.

Also Mumoli et al., (2014) emphasized on the nurses awareness about DVT diagnosis and investigation where he recorded that nurse-performed Compression ultrasonography (CUS) may be a potential useful alternative to physician performed CUS with a good accuracy.

\section{Recommendations:}

On the basis of the most important findings of the current study, the following recommendations are suggested:

- Designing an education programs and protocols about DVT prevention and it must be provided to health team members to be used as a reference guide in their performance.

- Integrating the DVT prevention programs and protocols into plan of 
Aida Faried Abd Elwanees Ali et. al.

care to replace the traditional nursing care plan.

\section{References:}

1. Elisha $\mathbf{S}$; Heiner $\mathbf{J}$; Nagelhout $\mathbf{J}$; Gabot M. (2015): Venous Thromboembolism: New Concepts in Perioperative Management. AANA Journal. 83(3):211-21.

2. Esmon, C. (2009): Basic mechanisms and pathogenesis of venous thrombosis. Blood Reviews, 23(5), 225-229.

DOI:

10.1016/j.blre.2009.07.002

3. Minet C, Potton L, Bonadona A, Roy RH, Somohano CA, Lugosi $M$, Cartier JC, Ferretti G, Schwebel C, Timsit JF. (2015): Venous thromboembolism in the ICU: main characteristics, diagnosis and thromboprophylaxis. Critical Care, 19:287.

4. McCaffrey R, Blum C. (2009): Venothrombotic Events: Evidencebased Risk Assessment, Prophylaxis, Diagnosis, and Treatment. Journal for Nurse Practitioners.5(5):325333. Elsevier Science, Inc

5. Leeper K. (2008): Venous Thromboembolism in the Intensive Care Unit Patient. NATF. Available at:

www.NATFonline.org/ethrombosis.php

6. Songwathana $\mathbf{P}$, Promlek $\mathbf{K}$, Naka K. (2010): Evaluation of clinical nursing practice guideline for preventing deep vein thrombosis in critically ill trauma patients. Australasian Emergency Nursing Journal. 13(4): 148.

7. Sole M L, Klein D G, Moseley M J. Introduction to critical care nursing. Six edition. Volume 1 .Philadephia:
Elsevier Saunders Inc, 2013; 423,

907.

8. Martin T, Oliver C. (2009): Prevention of deep vein thrombosis and pulmonary embolus. Anathesia and Intensive care medicine. 10:12. Elsevier Ltd.

9. Anthony M. (2013): Nursing assessment of deep vein thrombosis. MEDSURG Nursing. 22(2):95-8, 123.

10. Kahn RS, Shapiro S, Wells $\mathbf{S}$. (2013): Compression stockings to prevent post-thrombotic syndrome: a randomised placebo-controlled trial. Lancet. Available at: www.ncbi.nlm.nih.gov/pubmed.

11. Qaseem A, Snow V, Barry B. (2007): current diagnosis of venous thromboembolism in primary care. Clinical practice guidelines from American of Family Physician and American College of Physicians. Ann family Med. 5(1): 57-62.

12. Nacy D, Shebel N, Chyrie C, Whalen R. (2005): Diagnosis and management of iliac vein compression syndrome. Journal of vascular Nursing. 23(1): 10-17.

13. Scottish Intercollegiate Guidelines Network. (2013): Prevention and management of venous thromboembolism. Available at: www.sign.ac.uk/pdf/qrg122.

14. Geerts W and Selby R. (2003): Prevention of Venous Thromboembolism in the ICU. CHEST. 124:357-363.

15. Sud S, Mittmann N, Cook D, Geerts W, Chan B, Dodek P, Gould MK, Guyatt G, Arabi Y, Fowler RA. (2011): Screening and Prevention of Venous Thromboembolism in 
Critically Ill Patients. A decision analysis and economic evaluation. Am J Respir Crit Care Med. American Thoracic Society. 184: 1289-1298.

16. Fries D. (2011): Thrombosis prophylaxis in critically ill patients. Springer-Verlag. Wien Med Wochenschr. 161.3(4):68-72.

17. Bonner L; Johnson J. (2014): Deep vein thrombosis: diagnosis and treatment. Nursing Standard. 28(21):51-8; quiz 60.

18. Mumoli N; Vitale J; Cocciolo M; Cei M; Brondi B; Basile V; Sabatini S; Gambaccini L; Carrara I; Camaiti A; Giuntoli S; Dentali F.(2014): Accuracy of nurse-performed compression ultrasonography in the diagnosis of proximal symptomatic deep vein thrombosis: a prospective cohort study. Journal of Thrombosis \& Haemostasis. 12(4):430-5.

19. Kim H, Lee ES. (2014): Major difficulties and information needs recognised by nurses in applying graduated compression stocking and intermittent pneumatic compression for deep vein thrombosis prophylaxis. Journal of Clinical Nursing. 24: 308311. doi: 10.1111/jocn.12610.

20. oldhill D R, Badacsonyi

A, Goldhill A A, Waldmann C.(2008): A prospective observational study of ICU patient position and frequency of turning. Anaesthesia. 63: 509515.

21. Baidya S, Acharya $\mathbf{R}$ S, Coppieters M W.(2016):

Physiotherapy practice patterns in Intensive Care Units of Nepal: A multicenter survey.
Indian Journal of Critical Care Medicine. 20(2).

22. Yeole U L, Chand A C, Nandi B B, Gawali P P, Adkitte R G. (2015): Physiotherapy practices in Intensive Care Units across Maharashtra. Indian Journal of Critical Care Medicine. 19 (11):49-53.

23. Dafoe S, Chapman M J, Edwards S, Stiller K. (2015): Overcoming barriers to the mobilisation of patients in an intensive care unit. Anaesth Intensive Care. 43(6): 719-729.

24. Jesus F S D, Paim M D D, Brito J D O, Barros I D A, Nogueira T B, Martinez B P, Pires T Q. ( 2015): Mobility decline in patients hospitalized in an intensive care unit. Rev Bras Ter Intensiva. 28(2):114119. 